\title{
含有氢键供体大环化合物的构筑及其功能研究进展
}

\author{
黄国保 ${ }^{\dagger}, a$ 陈志林 ${ }^{\dagger}, a$ 韦贤生 ${ }^{a}$ 陈 钰 ${ }^{a}$ \\ 李秀英 $a$ 仲辉*, $b$ 谭明雄 $*, a$ \\ ( ${ }^{a}$ 玉林师范学院化学与食品科学学院 广西农产资源化学与生物技术重点实验室 广西玉林 537000) \\ ( ${ }^{b}$ 中国药科大学中药学院中药药理系 南京 211100)
}

\begin{abstract}
摘要 含有氢键供体基团构筑的大环化合物因其结构中具有可以提供氢键供体的 $\mathrm{N}-\mathrm{H}$ 基团，可以为大环化合物的主 客体化学提供额外的分子间作用力，在分子识别、自组装以及超分子催化等领域被广泛应用. 综述了近十年基于(硫) 脲键、酰胺键构筑的大环化合物的合成方法及其在分子识别中的最新研究进展. 为今后此类大环化合物的合成及应用 提供参考.
\end{abstract}

关键词 氢键供体基团; 大环化合物; 合成; 分子识别

\section{Recent Progress on the Construction and Function of Macrocyclic Compounds Containing Hydrogen Bond Donors}

\author{
Huang, Guobao ${ }^{\dagger, a} \quad$ Chen, Zhilin ${ }^{\dagger, a} \quad$ Wei, Xiansheng ${ }^{a} \quad$ Chen, $\mathrm{Yu}^{a}$ \\ Li, Xiuying $^{a} \quad$ Zhong, Hui $^{*, b} \quad$ Tan, Mingxiong*,a \\ ( ${ }^{a}$ Guangxi Key Laboratory of Agricultural Resources Chemistry and Biotechnology, College of Chemistry and \\ Food Science, Yulin Normal University, Yulin, Guangxi 537000) \\ ( ${ }^{b}$ School of Traditional Chinese Pharmacy, China Pharmaceutical University, Nanjing 211100)
}

\begin{abstract}
Because of the $\mathrm{N}-\mathrm{H}$ group structure in the macrocyclic compound containing hydrogen bond Donors, it can provide additional intermolecular forces in the host-guest chemistry, and this character is widely used in the molecular recognition, self-assembly, supramolecular catalysis and other fields. The recent progress on the synthetic methods of macrocyclic compounds based on (thio) urea, amide and its molecular recognition in $2010 \sim 2019$ are summarized. It is hoped that this review can be referred to synthesis and applications of this kind of macrocyclic compounds.

Keywords hydrogen bond donor group; macrocyclic compounds; synthesis; molecular recognition
\end{abstract}

大环化合物由于其特殊的环状分子结构及优良的 主客体性质, 成为超分子化学研究的主要内容之一 ${ }^{[1]}$. 自 1967 年 Pedersen ${ }^{[2]}$ 发现冠醚以来, 历时半个世纪的发 展, 大环化学的研究进入了突飞猛进的阶段. 早期基于 对冠醚、穴醚以及球醚的研究, 科学家们逐渐提出了超 分子化学的基本概念, 打破了传统化学的成键模式, 以 分子识别为基础, 分子组装为手段, 组装体功能为目标, 奠定了超分子化学与主客体化学的基础 ${ }^{[3]}$. 在超分子化 学发展的过程中, 先后出现了几代不同的主体代表的大
环化合物. 作为第一代超分子主体, 冠醚的研究仍然还 在进行 ${ }^{[4]}$. 继冠醚之后, 分别作为第二代和第三代超分 子主体, 环糊精 ${ }^{[5]}$ 和杯芳烃 ${ }^{[6]}$ 更进一步扩展了大环主体 的应用范围. 从第一代大环主体冠醚开始, 每一代大环 主体化合物的出现都为超分子化学引入了新的主-客体 体系，构筑了具有不同功能的复杂有序的超分子聚集 体.

鉴于大环受体的广阔应用前景, 对新型超分子大环 的探索也从未停止. 在近二十年的时间里, 很多新颖的

\footnotetext{
* Corresponding authors. E-mail: 1xya8401@163.com; tanmx00@163.com

Received September 18, 2019; revised October 31, 2019; published online December 2, 2019.

Project supported by the National Natural Science Foundation of China (No. 21961042), the Natural Science Foundation of Guangxi Province (No. 2018GXNSFAA294064) and the Yulin Normal University Research Grant (Nos. 2018YJKY36, 201810606010).

国家自然科学基金(No. 21961042)、广西自然科学基金(No. 2018GXNSFAA294064)和玉林师范学院科研(Nos. 2018YJKY36, 201810606010)资助项目.

共同第一作者(These authors contributed equally to this work).
} 
超分子大环化合物被报道出来, 并有很多具有非常独特 的性质 ${ }^{[7]}$. 这些大环化合物的合成与报道, 足以证明人 们对新型大环化合物的研究兴趣. 在被报道的新型大环 化合物中, 基于氢键供体基团构筑的大环化合物的研究 越来越火热. 因为这类大环化合物的环上含有氢键供体 基团, 可以提供额外的分子间的氢键作用力, 增加其分 子识别作用以及超分子催化性能. 对 2010 年至 2019 年 报道的基于氢键供体基团构筑的大环化合物进行了归 纳. 介绍了此类大环化合物的合成及分子识别与超分子 催化性能, 希望为今后基于氢键供体基团构筑的大环化 合物的合成与应用提供参考.

\section{1 基于(硫)胀键构筑的大环化合物}

对于(硫)脲基团而言, 其结构中含有两个 $\mathrm{N}-\mathrm{H}$ 基 团而具有一定的酸性, 与其它的超分子主体相比, 基于 (硫)嫝键构筑的大环化合物可以为主体提供额外的分子 间氢键, 以增强其分子识别, 自组装以及超分子催化特 性. 自 Shimizu 小组 ${ }^{[8]}$ 合成了基于双(硫)嫝键构筑的大环 化合物以来, 详细地研究了此类大环主体的超分子性 能 ${ }^{[9]}$, 并在此基础上衍生出(硫)脲键构筑的大环化合物 具有更加独特新颖的特性 ${ }^{[10]}$.

2011 年, Shimizu 课题组 ${ }^{[11]}$ 报道了利用 1,3-二乙炔
苯与过量的 4-碘苯甲醇作为反应原料, 经过四步有机反 应来构建含脲键大环分子 1 . 通过单晶衍射方法发现， 该大环化合物具有较大的空腔，分子内部的两个嫝基团 处于反平行状态, 且距离达到了 $1.8 \mathrm{~nm}$, 分子间通过嫝 基之间氢键的相互作用自组装形成圆柱状的纳米分子 管. Shimizu 小组利用得到的大环分子 1 作为催化剂, 在 紫外光照条件下可以使双分子香豆素发生光二聚反应， 得到具有高选择性的反式产物，并且使得该反应具有较 高的产率. 通过固态核磁、固态紫外分别对此催化反应 的机理做了比较详细的研究, 推测该催化反应中香豆素 分子进入了分子管内部, 在大环空腔以及䐖基的双重作 用下实现了较高的选择性合成(Scheme 1).

Shimizu 小组 ${ }^{[12]}$ 还利用吡啶代替苯环作为建筑单元 构建出含双嫝基大环分子 2. 通过单晶衍射(X-ray)分析, 这种大环分子可以自组装成无空腔的圆柱状结构. 与三 氟乙醇共结晶可以得到主客体复合物, 加热除去三氟乙 醇后，该大环分子 2 可以变形成为具有空腔的结构，并 且可以吸附氢气和二氧化碳等气体分子. 更为惊奇的 是，在客体诱导下得到的该主体大环可以与碘单质可逆 地形成一种稳定的主客体复合物. 实验结果通过单晶衍 射(X-ray)、光电子能谱(XPS)以及紫外可见光谱(UV)等 方法得到证实(Scheme 2).

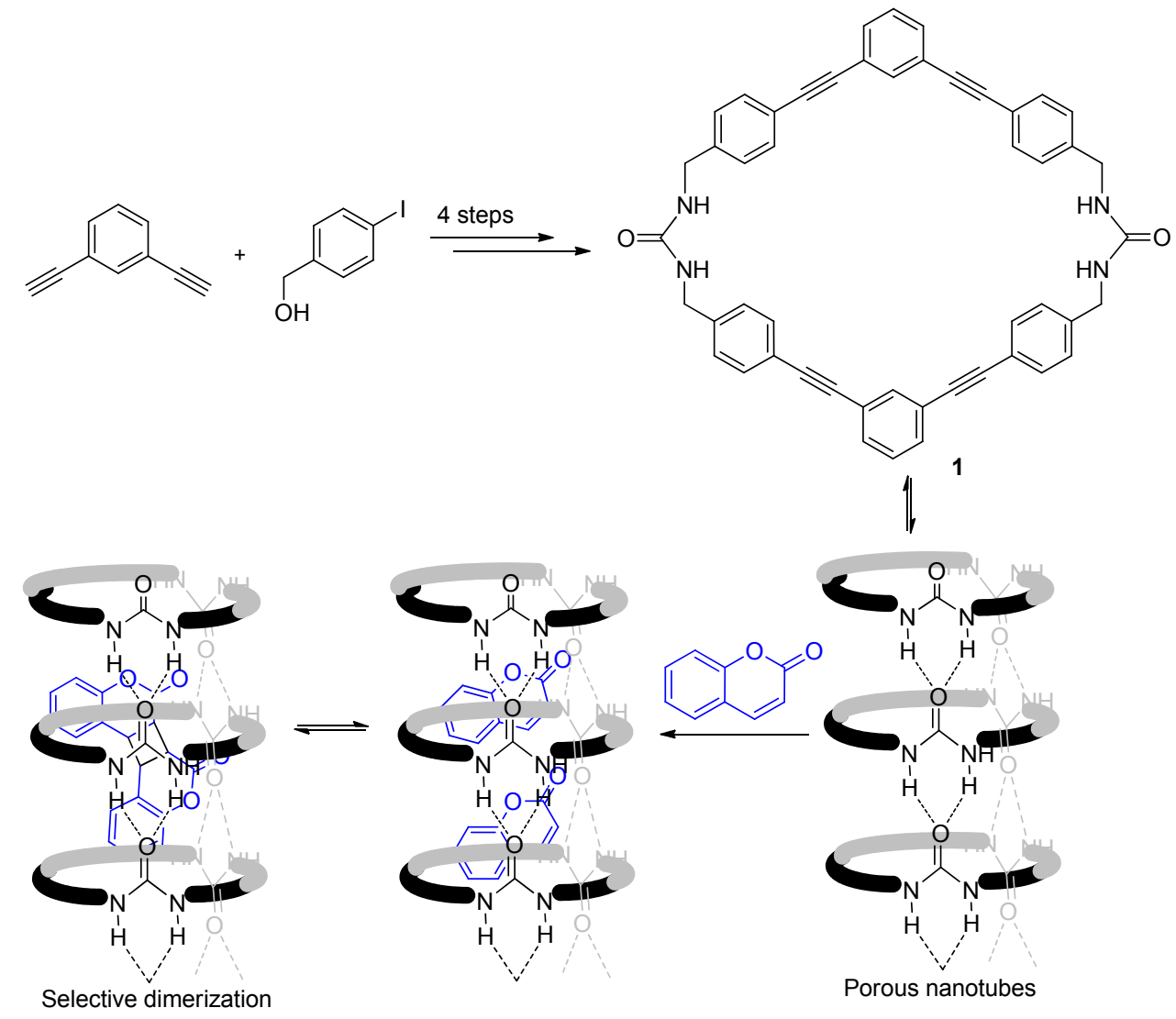

图式 1 含双腿基团的大环化合物 1 的合成及其催化反应

Scheme 1 Synthesis of the macrocycle 1 with bis-urea groups and its catalytic reaction 
<smiles>C=CCC</smiles><smiles></smiles>

图式 2 含双脲基团的大环化合物 2 的合成

Scheme 2 Synthesis of the macrocycle 2 with bis-urea groups

为了进一步探究结构单元与此类双腿大环分子的 结构之间的关系, Shimizu 课题组 ${ }^{[13]}$ 报道了基于萗环构 建的双腿大环分子 3 . 与基于苯环(吡啶环)构建的双嫝 分子不同的是, 䒺环之间呈现出一种独特的平行碗状结 构, 并且在不同的溶剂体系中得到两种不同的晶型 (Scheme 3).

吡啶作为建筑单元构建出含双腿基大环分子 $\mathbf{2}$, 可
以通过自组装形成紧密的一维圆柱状结构. 因为其内部 含有䐂基，氧原子上有孤对电子，可以作为分子间氢键 作用的受体. 2012 年, Shimizu 课题组 ${ }^{[14]}$ 利用大环主体 2 的这一特性将其用于三氟乙醇、苯酚、五氟苯酚以及乙 二醇等含有羟基类客体化合物的吸附. 主客体之间可以 通过分子间氢键作用形成非常有序的并且可逆的聚集 体. 不仅如此，大环分子 $\mathbf{2}$ 还可以与碘苯分子结合形成 有序的主客体复合物. 这一发现大大地增强了此类含嫝 大环的应用范围(Scheme 4).

2013 年, Shimizu 课题组 ${ }^{[15]}$ 将含嫝大环分子进行了 修饰，以二苯甲酮作为建筑基元在一定的条件下合成含 嫝大环分子 4 , 羰基的引入不仅使环的空腔直径增大到 约 $0.7 \mathrm{~nm}$, 而且衍生出新的催化性能. 通过单晶衍射验 证了大环分子 4 通过分子间的氢键以及芳环之间的 $\pi-\pi$ 堆积作用形成稳定的分子管构型，2-甲基-2-丁烯和异丙 苯等客体分子可以进入空腔内部. 在紫外光照射以及有 氧环境下选择性氧化得到对应的醇(Scheme 5).

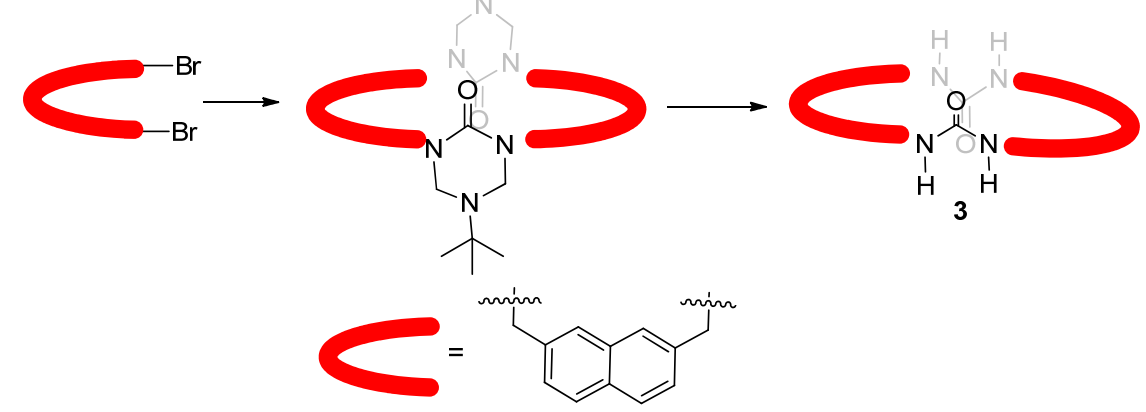

图式 3 含双脲基团的大环化合物 3 的合成

Scheme 3 Synthesis of the macrocycle 3 with bis-urea groups

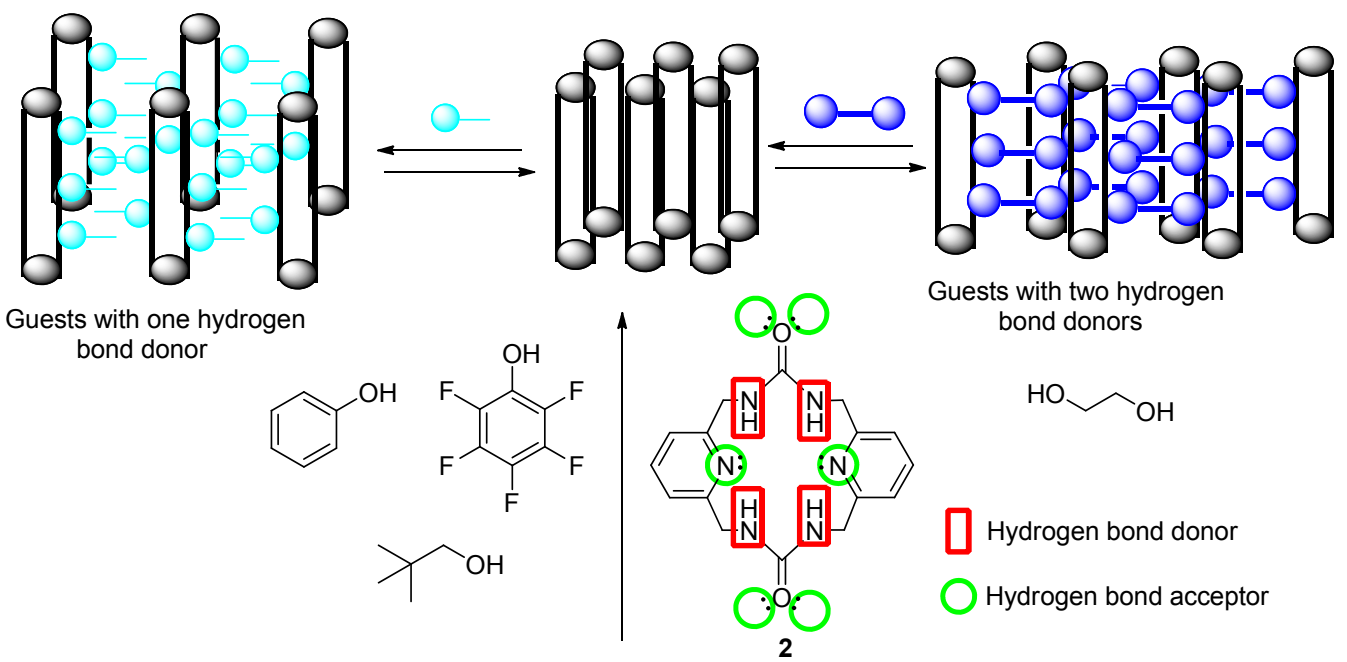

图式 4 大环化合物 2 与不同醇分子的组装

Scheme 4 Assembly of the macrocycle 2 with different alcohol molecules 

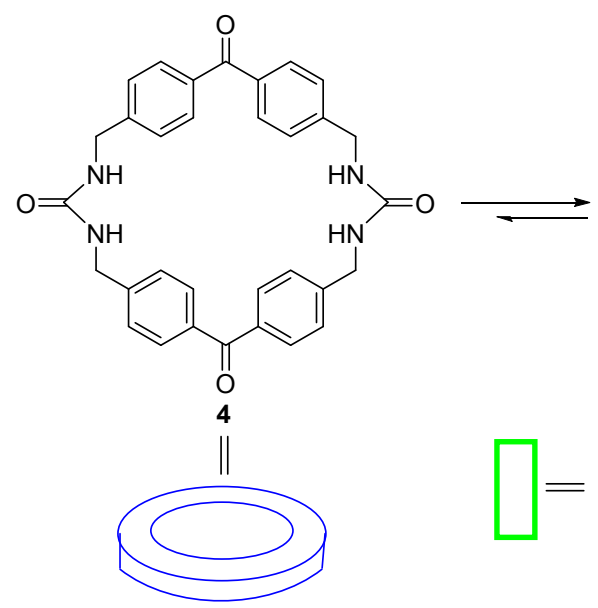
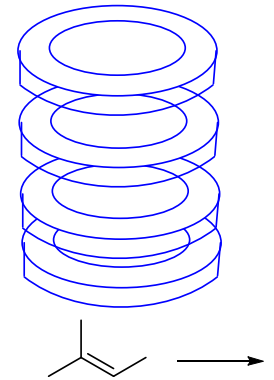

$\curvearrowright \mathrm{NH}_{\mathrm{OH}}$
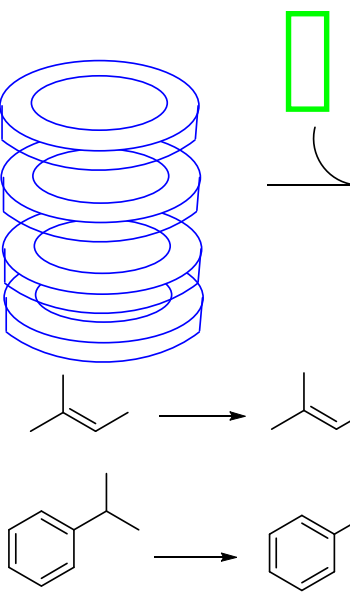<smiles>CC(C)(O)c1ccccc1</smiles>

$63 \%$ conversion $60 \%$ selectivity

图式 5 大环化合物 4 参与的选择性氧化反应

Scheme 5 The macrocycle 4 participate in selective oxidation reactions

2013 年, 王乐勇课题组 ${ }^{[16]}$ 基于二芳基乙烯为建筑 单元, 分别经过多步合成反应得到了两种含脲大环分子 $\mathbf{5 a} / \mathbf{5 b}$, 该小组还通过紫外一可见光谱详细研究了二者的 光致变色行为, 并在分子的结构上对实验结果进行了具 体分析. 大环化合物 5a 相对 $\mathbf{5 b}$ 而言, 因为空腔较小约 束了分子内的构象变化, 从而 $5 \mathbf{b}$ 体现出较好的光致变 色现象. 遗憾的是没有得到两种大环分子的单晶结构, 只能从其前体的单晶构型以及核磁共振谱来推测分子 结构.
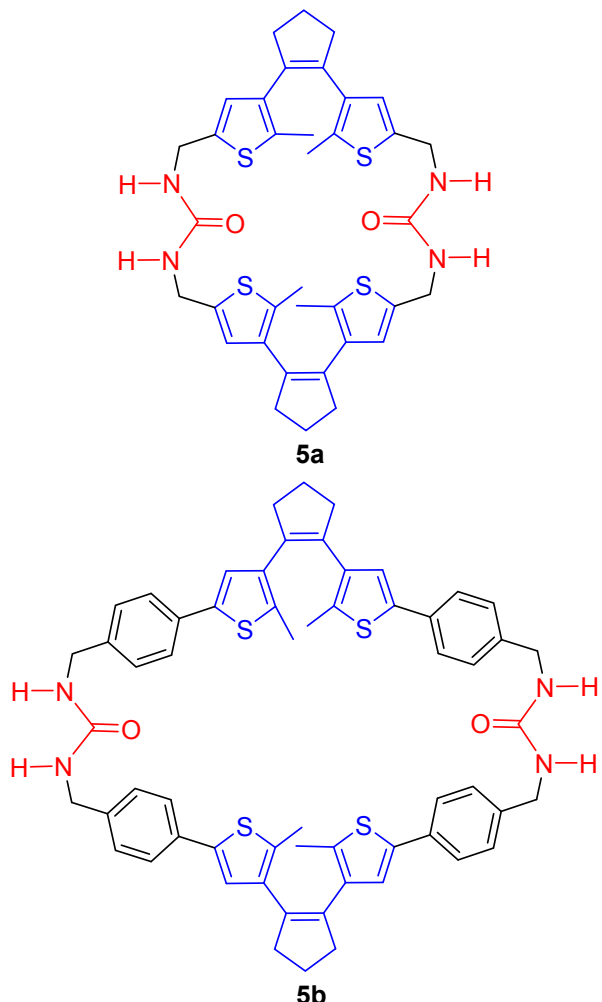

2013 年, 陈金平课题组 ${ }^{[17]}$ 利用羰基二咪唑和氨基
高效合成脲基团的反应以及三聚氯氰分步取代反应的 特点，通过片段偶联法方便地合成骨架中含有双脲基 团一氧杂大环化合物 $6 \mathrm{a}$, 分步产率达到 54\%. 通过对大 环侧链的修饰改造得到 $6 \mathbf{b} / 6 \mathbf{c}$, 并运用吸收光谱和核磁 共振方法研究了它们与卤素阴离子的相互作用, 得到的 大环化合物可与卤素阴离子以 $1: 1$ 模型结合, 结合常 数达到 $10^{5} \sim 10^{8} \mathrm{~L} \cdot \mathrm{mol}^{-1}$, 结合强度顺序为: $\mathrm{F}^{-}>\mathrm{Cl}^{-}>$ $\mathrm{Br}^{-}>\mathrm{I}^{-}$.

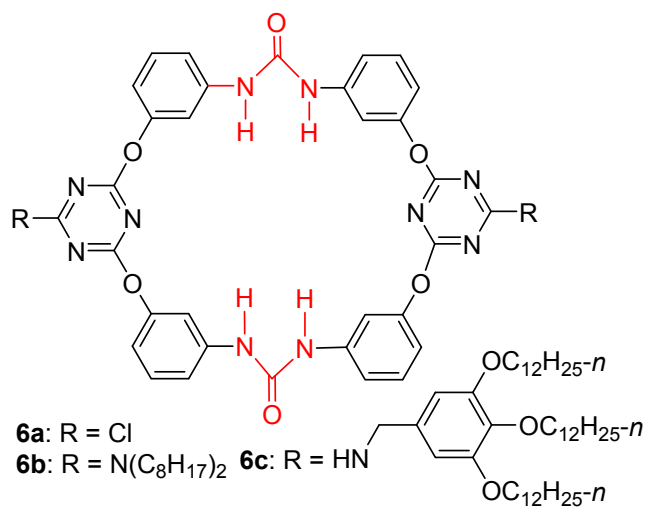

2014 年, Beck 课题组 ${ }^{[18]}$ 构建了基于二苯乙炔二胺 类衍生物与嶡基二咪唑两个含脲大环分子 $7 \mathrm{a} / 7 \mathbf{b}$, 具有 较高的收率(均达到了 $60 \%$ ), 而且反应的后处理简单, 产物易于分离. 在高温 $\left(T>130{ }^{\circ} \mathrm{C}\right)$ 真空升华下 $7 \mathrm{a}$ 可以 转化成吲哚-喹啉酮. 相比较 7a 而言, 因为环上多了炔 基，导致 7b 具有更大的空腔. 在极性溶剂 $N, N$-二甲基 甲酰胺(DMF)或者二甲基亚砜(DMSO)的环境中，可以 得到 $7 \mathbf{b}$ 与溶剂分子复合物的单晶结构. 从单晶结构中 发现，大环呈现近平面结构，分子中双䐂基(与平面夹 角 $28^{\circ}$ )上的 $\mathrm{N}-\mathrm{H}$ 均指向空腔内部, 可以作为较好的氢 键供体与富电子的原子或离子结合. 该小组通过核磁共 
振氢谱, 详细研究了两种大环分子与常见的阴离子的键 合作用, 发现大环分子均可以与阴离子有键合作用. 其 中大环分子 $7 \mathrm{~b}$ 与氯离子 $\left(\mathrm{Cl}^{-}\right)$具有较强的分子间作用 (Scheme 6).<smiles>CC#Cc1ccccc1C#C[C+]=Nc1ccn(C(=O)n2ccnc2)c1</smiles>

$n=0,1$<smiles>C#CC#Cc1ccccc1NC(=O)Nc1ccccc1C#CC#Cc1ccccc1NC(=O)Nc1ccccc1C#C</smiles>

$7 a n=0$
图式 6 含嫝大环化合物 $7 \mathrm{a} / 7 \mathrm{~b}$ 的合成

Scheme 6 Synthesis of the macrocycles $7 \mathbf{a}$ and $7 \mathbf{b}$ with bis-urea groups

2015 年, 蒋伟课题组 ${ }^{[19]}$ 以 2,6-二羟基菜作为反应原 料, 经过多步反应合成两种构型异构的含嫝大环分子 $\mathbf{8 a} / \mathbf{8 b}$. 产物的结构通过二维核磁以及单晶衍射得到证 实. 与之前其他课题组合成的含脲大环不同的是，这两 种大环分子具有较大且较深的空腔，并且脲基中的 $\mathrm{N}-$ $\mathrm{H}$ 均指向空腔内部(图 1). 无论是反式构型的 8a 还是顺 式构型的 $8 \mathrm{~b}$, 都可以与乙腈溶剂分子通过分子间的氢 键作用形成较好的主客体复合物。受此实验现象的启 发, 该小组用核磁共振方法详细研究了这两种含脲大环 与 1,4-二氧六环、吡嗪、三乙烯二胺等有机小分子的识 别性能. 在氯仿的非极性环境下，这些有机小分子均可 以进入大环空腔内部, 通过环上 $\mathrm{N}-\mathrm{H}$ 与客体中的氢键 受体原子结合形成稳定的主客体复合物, 其中反式构型 大环 $8 \mathbf{a}$ 与三乙烯二胺的键合能力最强.

蒋伟课题组继续研究了此类大环分子. 2016 年, 该 小组 ${ }^{[20]}$ 对大环上的氢键供体基团进行了修饰，得到了 含双硫嫝的大环分子 $9 a / 9 b$, 并详细研究了四种大环分 子的分子识别性能. 相比较含嫝大环 $8 \mathbf{a} / \mathbf{8 b}$, 含硫脲的 大环分子 $9 a / 9 b$ 具有更强的氢键供体能力, 在主客体作 用中体现出与客体具有更强的键合作用。
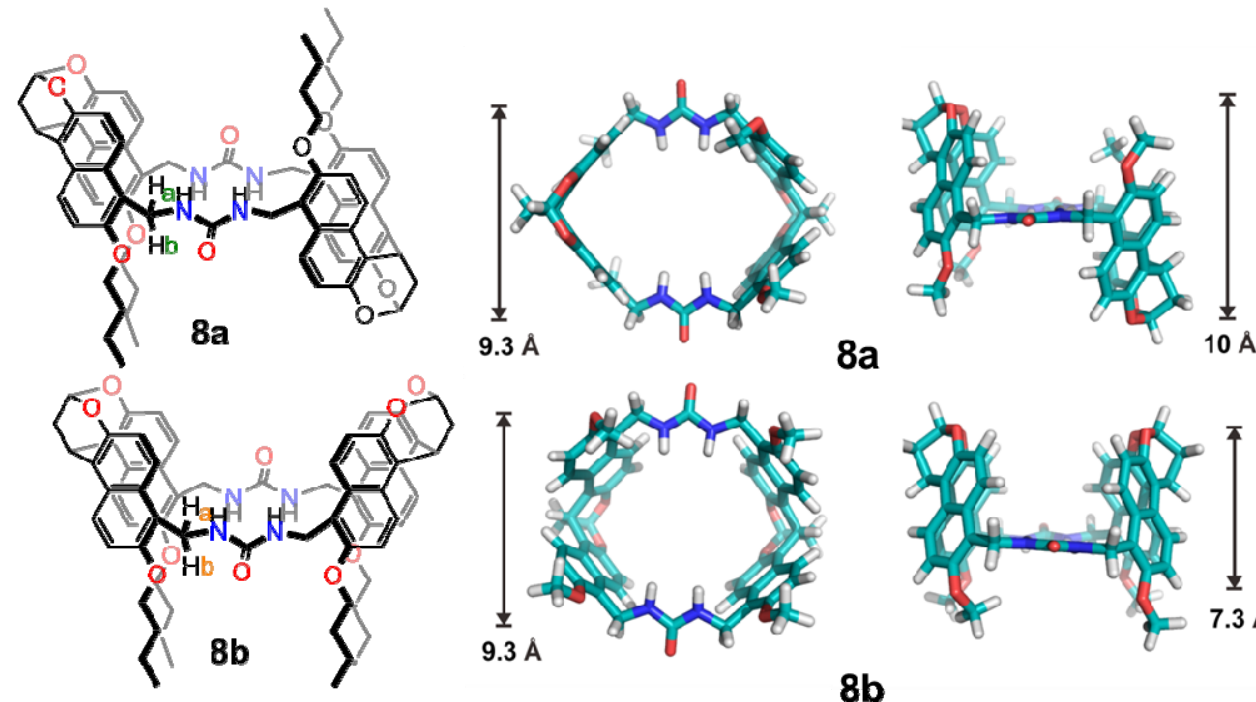
$10 \AA$

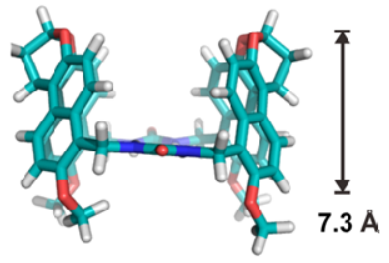

$8 \mathbf{b}$

图 1 含腿大环化合物 $\mathbf{8 a} / \mathbf{8 b}$ 的结构

Figure 1 Structure of macrocycles $8 \mathbf{a}$ and $\mathbf{8 b}$ with bis-urea groups
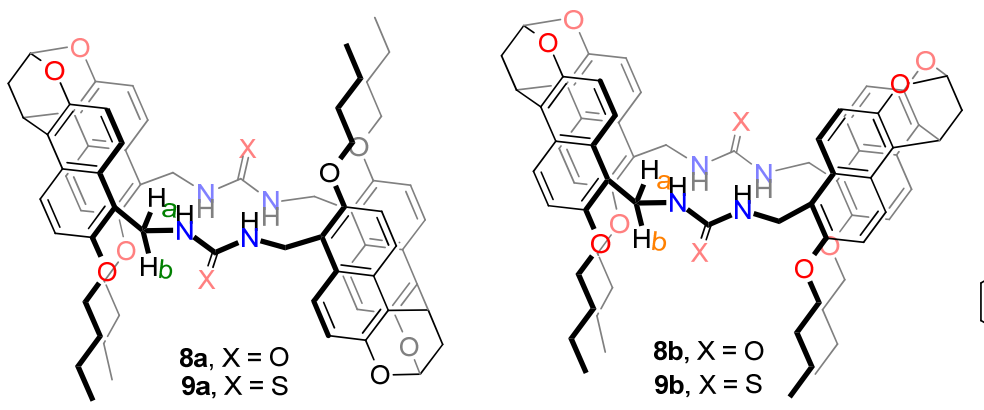<smiles>C1COCCO1</smiles><smiles>c1cnccn1</smiles><smiles>Cc1nccnc1C</smiles><smiles>C1CN2CCN1CC2</smiles><smiles>C1COCOC1</smiles><smiles>c1ccncc1</smiles><smiles>c1cncnc1</smiles><smiles>C1CN2CCC1C2</smiles><smiles>O=[N+]([O-])c1ccccc1[N+](=O)[O-]</smiles>

图 2 含嫝大环化合物 8 和 9 与不同客体的识别

Figure 2 Recognition of urea macrocyclic compounds $\mathbf{8}$ and $\mathbf{9}$ with different guests 
不仅如此，该小组 ${ }^{[21]}$ 还发现这四种大环分子还可以与 芳香族化合物通过分子间的 $\mathrm{C} / \mathrm{N} 才 \mathrm{H} \cdots \pi$ 相互作用得到 较好的识别性能.

Kondo 课题组 ${ }^{[22]}$ 基于联荎为建筑基元合成的双艮 大环分子 10 与氯离子 $\left(\mathrm{Cl}^{-}\right)$具有较好的分子识别作用, 并从其主客体复合物的单晶结构中发现存在两种对映 体构型. 2019 年, Kondo 小组 ${ }^{[23]}$ 详细研究了大环化合物 10 与四种不同的手性羧基阴离子的识别行为(图 3). 先 通过紫外-可见滴定法(UV-vis titrations)确定了主客体 复合物的键合计量比为 $1: 1$. 该大环分子结构中脲基 的四个 $\mathrm{N}-\mathrm{H}$ 基团以及主客体间的 $\mathrm{CH}-\pi$ 协同作用下表 现出较好的分子识别行为, 利用核磁滴定 ${ }^{1} \mathrm{H}$ NMR titrations)法测得大环主体与手性羧基阴离子 $\mathrm{Ac}^{-} \mathrm{LeuO}^{-}$具 有较好的识别能力. 在此基础上也运用了圆二色谱滴定 法(CD spectral titrations)详细研究了大环分子 $\mathbf{1 0}$ 与手性 羧基阴离子 $\mathrm{Ac}-\mathrm{LeuO}^{-}$客体的分子识别. 并且通过密度 泛函理论(Cartesian coordinates of DFT calculations)计算 得出大环分子逆时针的扭曲构象是影响不对称识别的 主要因素. 此研究中的大环分子可以作为一种很好的手 性分子探针，通过与客体分子间的氢键作用实现手性诱 导和检测.
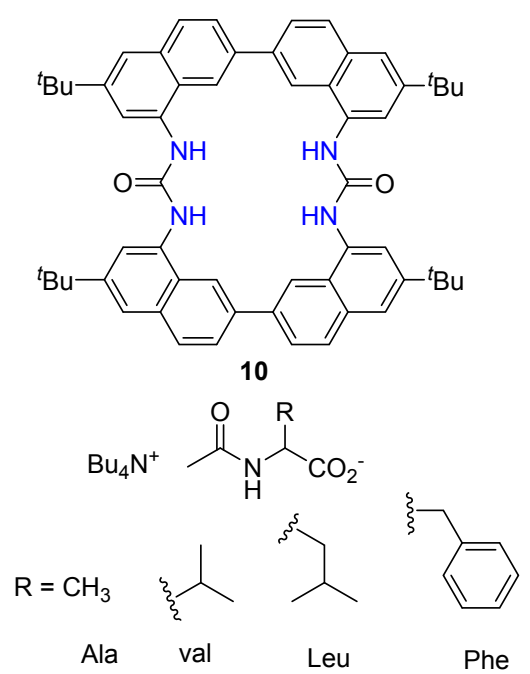

图 3 含嫝大环化合物 $\mathbf{1 0}$ 与不同客体分子的识别

Figure 3 Recognition of urea macrocyclic compound $\mathbf{1 0}$ with different guests

2019 年, Davis 课题组 ${ }^{[24]}$ 报道了基于脲基构建的水 溶性分子笼 11 , 在水相环境中实现对 $\beta$-呋喃糖苷类有机 物较好的识别作用. 分子笼 11 的分子结构中含有 6 个脙 基, 并且其中的 $\mathrm{N}-\mathrm{H}$ 均指向分子内部. 其侧链( $\mathrm{R}$ )修饰 了具有多个 “树枝状” 的水溶性基团, 以增强分子的水 溶性. 在分子间氢键以及 $\mathrm{C}-\mathrm{H} \cdots \pi$ 等多重分子间作用 力下, 分子笼 11 与葡萄糖的特异性识别作用达到了分 子生物水平(键合常数 $K=18000 \mathrm{~L} \cdot \mathrm{mol}^{-1}$ ). 这一发现对
糖尿病人的治疗具有积极的意义.
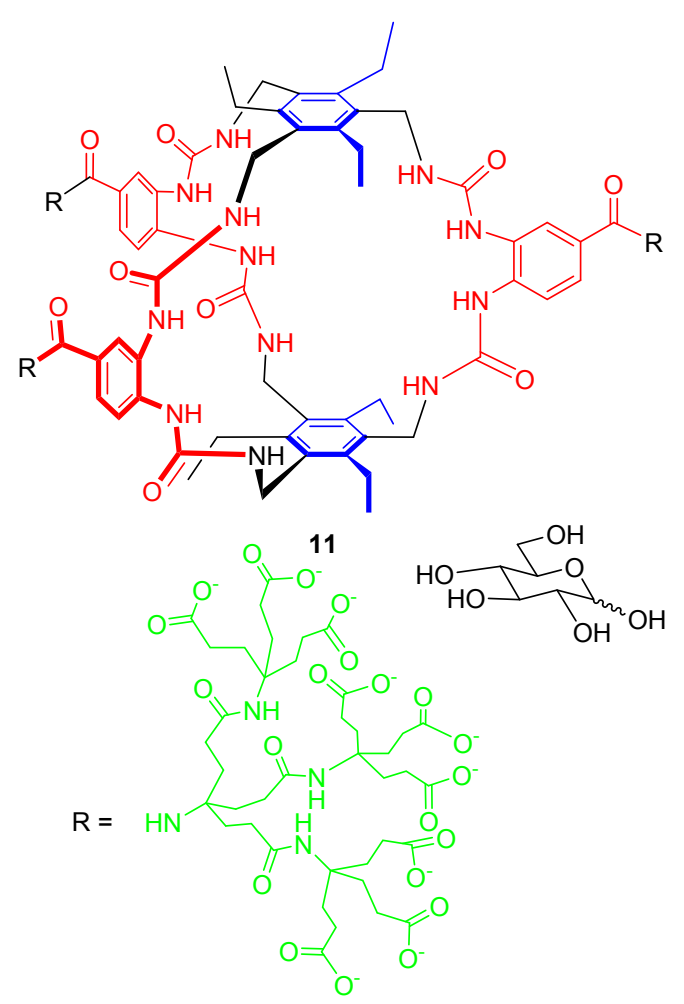

图 4 含嫝大环分子 11 与葡萄糖分子的识别

Figure 4 Recognition of urea macrocyclic compound $\mathbf{1 1}$ with glucose

\section{2 基于酰胺键构筑的大环化合物}

近年来, 由酰胺键构建的许多新型大环分子因其独 特的分子识别能力和优良的超分子催化性能被国内外 学者广泛报道 ${ }^{[25]}$. 与(硫)䐂基团相似, 酰胺基团中也含 有 $\mathrm{N}-\mathrm{H}$ 键, 同样可以作为氢键的供体. 研究酰胺键构 建的大环分子与不同类型的客体分子之间的分子作用， 越来越受到关注，早期以 Leigh 小组 ${ }^{[26]}$ 、Davis 小组 $^{[27]}$ 等的研究较为深入. 他们不仅合成了多种基于酰胺键构 建的新型大环分子, 还在此基础上详细研究了其分子识 别及自组装行为.

Zeng 小组 ${ }^{[28]}$ 在基于酰胺键构建的大环化合物方面 也有独到的研究, 构建出由酰胺键连接的芳环五聚体大 环分子, 并通过单晶衍射研究了大环的分子结构, 空腔 内部的酰胺 $\mathrm{N}-\mathrm{H}$ 与甲氧基上的 $\mathrm{O}$ 原子形成了网状的氢 键, 稳定了其 “平面圆盘式” 的构型. 2011 年, 该小组 ${ }^{[29]}$ 继续研究了这类五聚体大环分子性能的研究. 在之前的 工作基础上改变大环分子空腔内部的甲氧基的数目和 位置, 精准地调控大环分子的结构和分子识别行为. 为 了研究这种大环分子在溶剂相中的分子识别性能, 在其 空腔外部引入了较长的烷基链以增强其溶解度. 在去质 子化作用下, 通过等温量热滴定法(ITC)详细研究了大 
环主体阴离子与不同碱金属离子的键合能力 $(13,13 \mathrm{~b} \sim$ 15b). 单晶结构也证实金属离子进入空腔内部形成稳定 的主客体复合物.
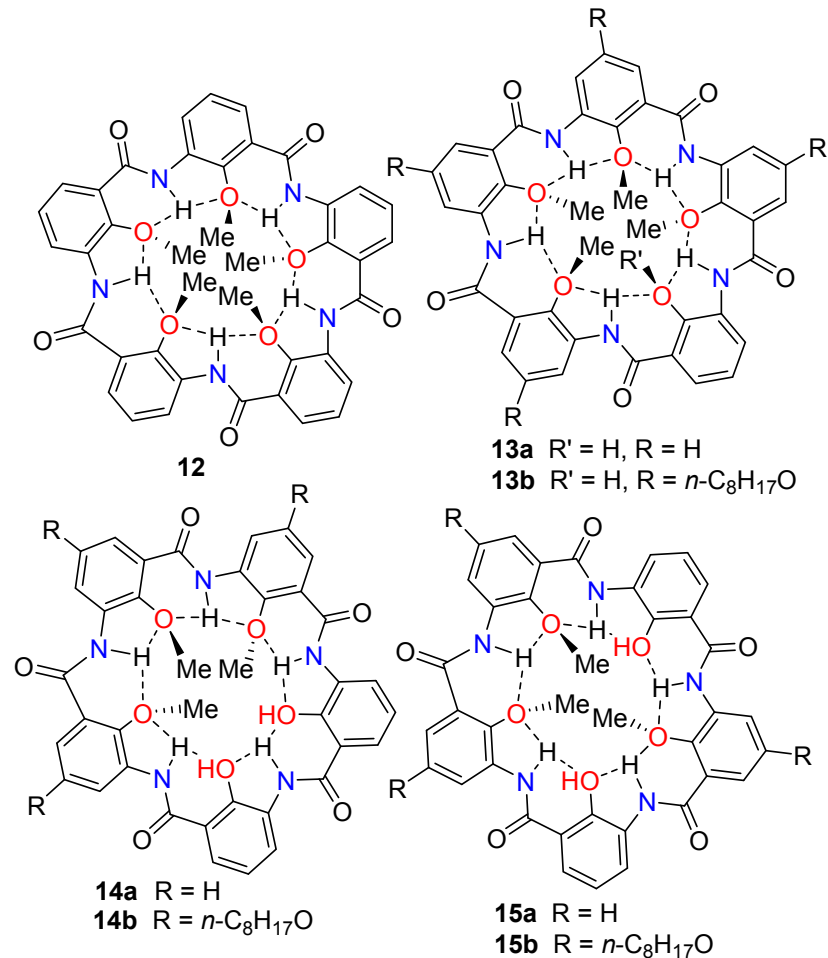

Gong 小组 ${ }^{[30]}$ 合成了由酰胺键连接的芳基的六聚体 大环分子, 并研究了其自组装行为. 2011 年, 该小组 ${ }^{[31]}$ 通过改变建筑单元的侧链取代基团, 分别合成出大环分 子 16. 在核磁共振氢谱中, $16 \mathbf{a}$ 在 $\mathrm{CDCl}_{3}$ 的溶剂环境中 很难观察到 $\mathrm{H}$ 的化学位移, 即使升高温度至 $50{ }^{\circ} \mathrm{C}$ 也不 能明显观察到分子中 $\mathrm{H}$ 的峰形. 这在一定程度上说明了 因为分子自组装导致了 $16 \mathbf{a}$ 环上的 $\mathrm{H}$ 不能够自由交换. 通过侧链的修饰, $16 \mathrm{~b} / 16 \mathrm{c}$ 在相同溶剂环境下的核磁共 振氢谱才逐渐清晰, 该大环分子的核磁结果受到环境极 性以及自身浓度的影响较为明显. 动态光散射(DLS)详 细研究了大环分子的自组装行为, 在氯仿环境中, 随着 $16 \mathrm{~b}$ 的浓度从 $0.25 \mathrm{mmol} / \mathrm{L}$ 增大到 $5 \mathrm{mmol} / \mathrm{L}$, 其聚合长 度也从 $550 \mathrm{~nm}$ 增长到 $1909 \mathrm{~nm}$. 在浓度为 $9 \mathrm{mmol} / \mathrm{L}$ 时 增长到 $2045 \mathrm{~nm}$ 并趋近于平稳. 不仅如此, 核磁共振氢 谱证实, 大环分子的自组装行为主要是通过分子间的偶 极作用以及 $\pi-\pi$ 堆积共同作用的结果. 同时也运用扫描 电镜(SEM)、透射电镜(TEM)、原子力显微镜(AFM)以 及 $X$ 射线粉末衍射 $(X R D)$ 等技术研究了大环分子的自组 装行为.

Davis 小组 ${ }^{[32]}$ 在基于酰胺键构建大环分子方面的研 究持续火热. 他们多次报道了合成的含酰胺键大环分子 可以较好地识别一系列糖类. 2011 年, 该小组 ${ }^{[33]}$ 在之前 的研究基础上改变了建筑基元, 构建了基于联苯的酰胺

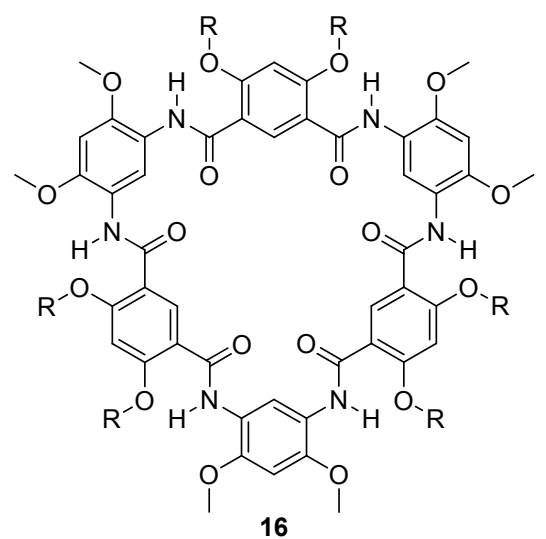

16a: $\mathrm{R}=\left(\mathrm{CH}_{2}\right)_{7} \mathrm{CH}_{3}$ 16b: $\mathrm{R}=\left(\mathrm{CH}_{2}\right)_{9} \mathrm{CH}=\mathrm{CH}_{2}$ 16c: $\mathrm{R}=\left(\mathrm{CH}_{2}\right)_{11} \mathrm{CH}_{3}$ 16d: $\mathrm{R}=\mathrm{CH}_{3}$

大环分子 $17 \mathbf{a} / \mathbf{1 7 b}$. 通过改变联苯的取代基团, 详细研 究了分子间的 $\mathrm{C}-\mathrm{H} \cdots \pi$ 作用对主客体的分子识别影响. 他们发现此类大环分子与糖类识别作用是分子间氢键, 主客体间 $\mathrm{C}-\mathrm{H} \cdots \pi$ 作用以及大环分子的疏水性能共同 作用的结果. 在随后的十年间, Davis 小组 ${ }^{[34]}$ 不断通过改 变不同的含有大 $\pi$ 键的建筑基元以增强主客体间 $\mathrm{C}-$ $\mathrm{H} \cdots \pi$ 作用，合成了多个基于酰胺键构建的大环化合物 分子, 并详细研究了在水相环境中的主客体化学, 特异 性地识别不同的单糖、二糖以及多糖类客体分子, 取得 了较好的研究成果.

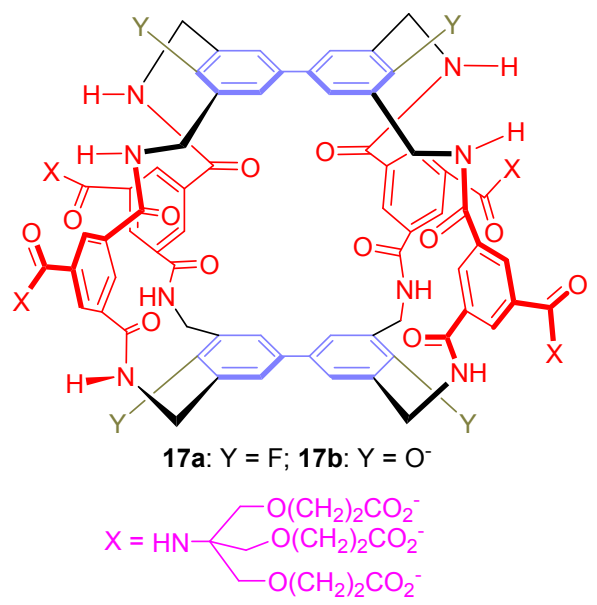

蒋腊生课题组 ${ }^{[35]}$ 报道了吡啶 $N$-氧化物作为客体与 二酰胺大环化合物 18/18b 的相互作用, 实验结果表明, 主客体分子之间以 $1: 1$ 络合形成准轮烷结构, 并通过 酸碱调控实现分子开关功能. 2012 年，该小组通过吡啶 二酰胺大环与碘甲烷反应，合成了一种阳离子型大环主 体化合物 19, 并用 NMR, ESI-MS 进行了结构表征. 采 用 ESI-MS 和 ${ }^{1} \mathrm{H}$ NMR 方法研究了合成的大环化合物与 吡啶 $N$-氧化物客体的相互作用, 通过核磁滴定测定了 主客体络合物的络合常数 ${ }^{[36]}$. 实验结果表明，阳离子型 
大环化合物 19 不仅能与吡啶 $N$-氧化物形成 $1: 1$ 准轮烷 型络合物, 而且其络合常数 $K_{\mathrm{a}}$ 比相应的中性大环化合 物 $18 b$ 有显著提高.

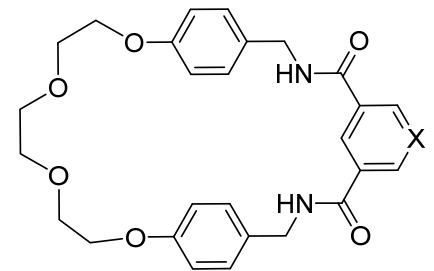

18a: $X=\mathrm{CH} ; 18 b: X=N$<smiles>CCCCCNC(=O)c1cc(C(=O)NCCCC)c[n+]([O-])c1</smiles><smiles>C[n+]1cc2cc(c1)C(=O)NCc1ccc(cc1)OCCOCCOCCOc1ccc(cc1)CNC2=O</smiles><smiles>O=C(NCc1ccccc1)c1cc(C(=O)NCc2ccccc2)c[n+]([O-])c1</smiles>

2013 年, 黎占亭课题组 ${ }^{[37]}$ 以吡啶二胺与 1,3 -二异丁 基苯作为反应原料, 经过多步有机反应通过 1,3-偶极环 加成反应合成出了含有 1,2,3-三唑的酰胺键大环 20a/20b. 经过单晶衍射研究发现: 无论是大环分子 20a, 还是具有更大空腔结构的 $20 b$, 分子内三唑上的 $\mathrm{C}-\mathrm{H}$ 与苯环侧链的 $\mathrm{O}$ 原子通过氢键作用使得大环的构型稳 定. 通过核磁滴定法, 苂光滴定法详细研究了此类大环
分子与酰胺类，单糖类以及卤化物等不同客体分子在二 氯甲烷，氯仿中的分子识别行为，证实无论是哪一种客 体分子, 与大环主体上三唑上的 $\mathrm{N}$ 原子之间的作用力比 较微弱，只有在多重分子间的氢键作用下主客体复合物 才能够稳定. 说明分子间的氢键作用是影响主客体识别 的主要因素. 该小组后续还研究并合成了含有酰胺与腙 共同构建的大环分子 ${ }^{[38]}$.

受到 Glass 合成的双酰胺键大环分子 ${ }^{[25 a]}$ 的启发，蒋 伟课题组 ${ }^{[39]}$ 在此基础上做了更加细致的研究. 2016 年, 该小组报道了利用合成的两种具有不同构型的水溶性 双酰胺键大环分子 $\mathbf{2 1 a} / \mathbf{2 1 b}$, 在水相环境中实现了对 1,4-二氧六环分子的特异选择性识别，通过核磁滴定 法、苂光滴定法以及恒温滴定量热法(ITC)详细研究了分 子间的识别行为(图 5). 研究发现不同构型的大环分子 对 1,4-二氧六环的键合能力不同, 顺式构型的大环 21a 具有与客体更强的键合能力, 对 1,4-二氧六环的键合常 数达到了 $13500 \mathrm{~L} \cdot \mathrm{mol}^{-1}$.

2016 年, Luis 课题组 ${ }^{[40]}$ 报道了基于阴离子模板效应 高效合成酰胺键大环. 该小组以缩氨酸衍生物 22-1 与 间苯二甲酰亚胺 22-2 为反应底物，在二氯甲烷的溶剂 环境中加入不同的阴离子作为模板 $\left(\mathrm{Cl}^{-}, \mathrm{Br}^{-}, \mathrm{I}^{-}\right)$合成大 环 22a/22b) (Scheme 7). 实验结果发现: 无论是哪一种 缩氨酸衍生物作为反应底物, 加入氯离子作为模板的反 应可以较高产率的得到相应的酰胺键大环 22a. 这种合

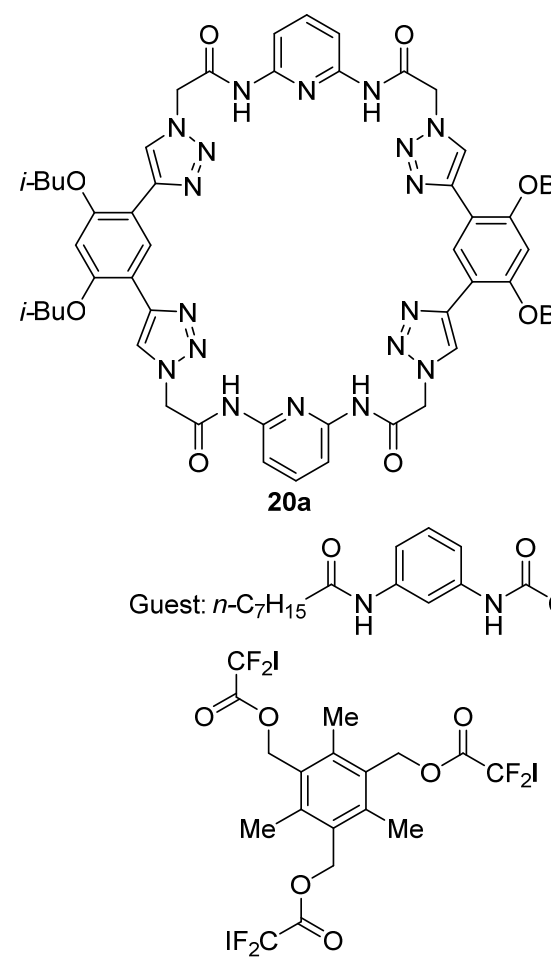

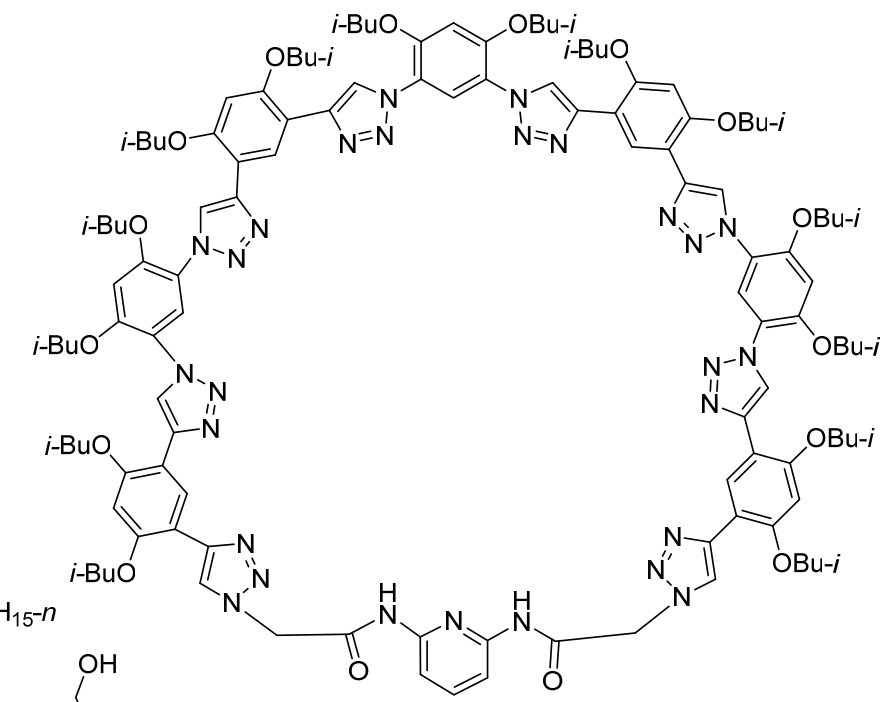<smiles>OCCOC1C(CO)OC(O)C1O</smiles>

图 5 大环化合物 $20 \mathrm{a} / 20 \mathrm{~b}$ 与不同的客体分子

Figure 5 Macrocycles 20a/20b and different guests 

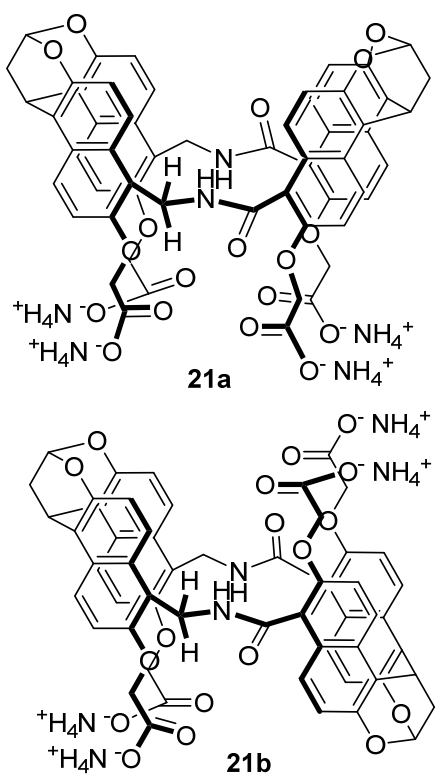

成方法为酰胺键大环的合成提供了一条可借鉴的实验 方法.

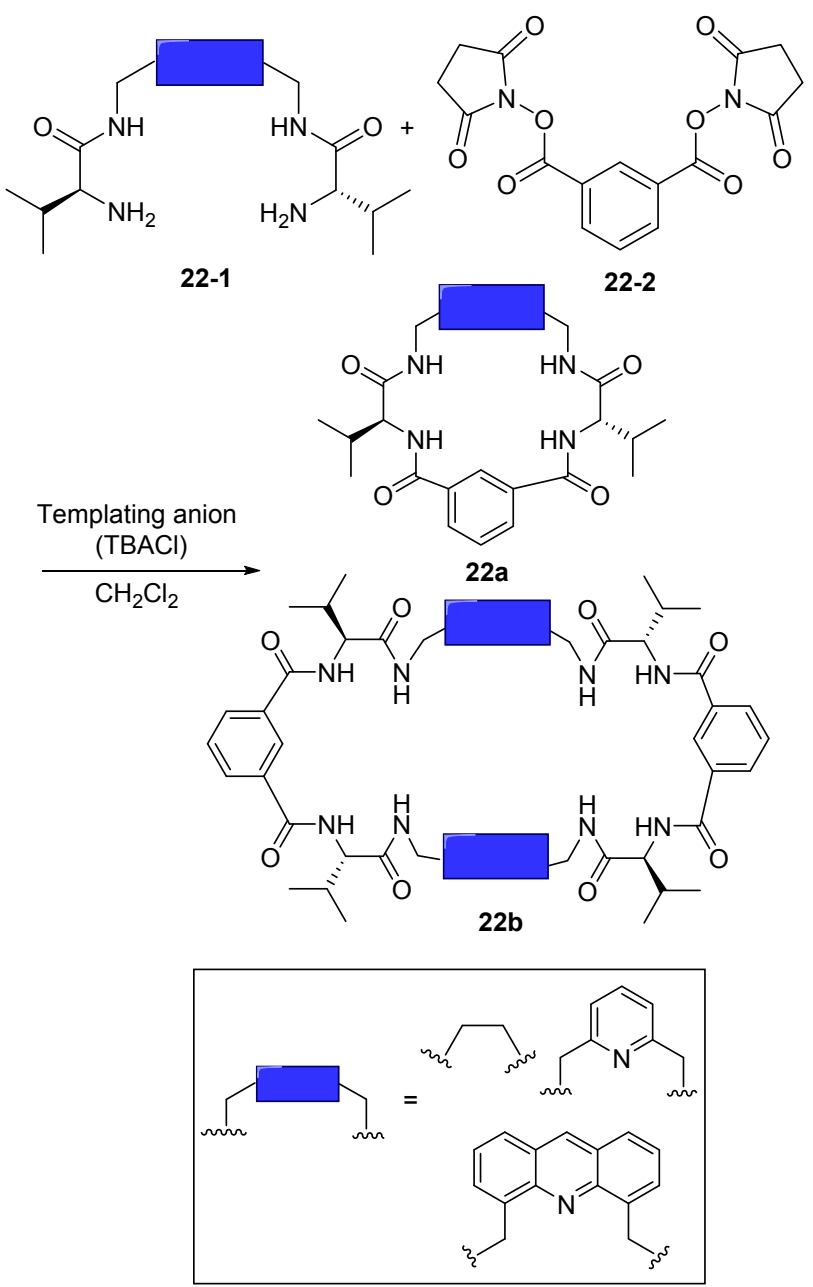

图式 7 氯离子模板效应合成大环化合物 22a/22b

Scheme 7 Macrocycles 22a and 22b synthesized by chloridetemplated amide bond formation
2017 年，袁立华课题组 ${ }^{[41]}$ 构建含有 6 个酰胺键的大 环分子 23, 并详细研究了与不同的咪唑盐类客体的分 子识别性能. 大环分子 $\mathbf{2 3}$ 的空腔直径达到 $0.817 \mathrm{~nm}$, 可 以较好的包结咪唑环 $(0.45 \mathrm{~nm}$ )(图 6). 改变咪唑环上的 取代基的大小或者抗衡的阴离子，二者的主客体复合物 能够较好地形成稳定的离子液体. 通过改变体系的浓 度，竞争离子以及体系的酸碱度，可以实现对这种复合 物离子液体的临界溶液温度的精准控制. 更为有趣的 是, 大环分子 23 与碘离子咪唑环形成的主客体复合物 可以分离两种不同的有机染料, 加深了超分子复合物的 应用范围。

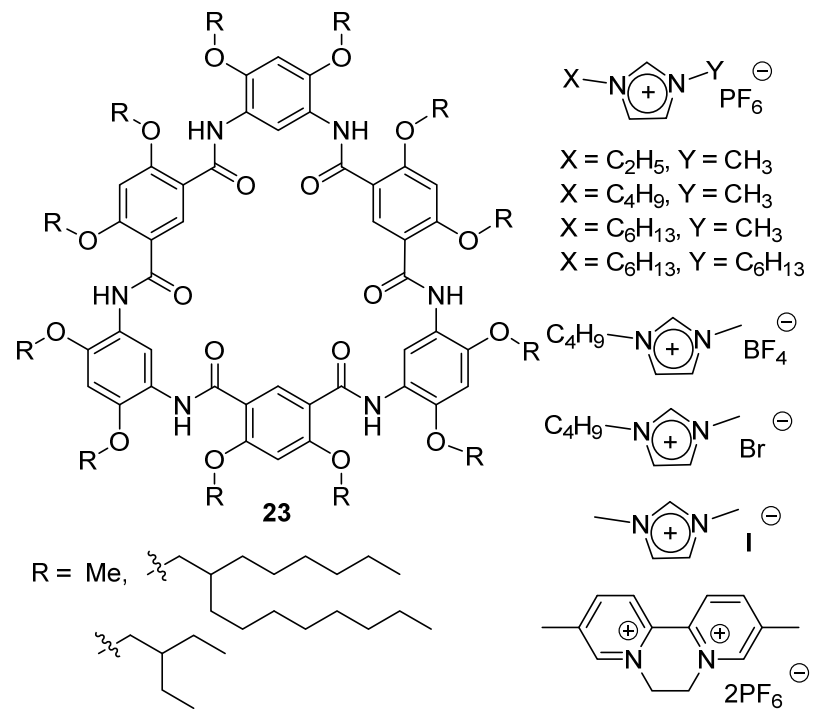

图 6 大环化合物 23 与不同的客体分子

Figure 6 Macrocycle 23 and guests

朱必学课题组 ${ }^{[42]}$ 一直致力于酰胺大环化合物的合 成及其阴离子识别性能研究. 2018 年, 该小组 ${ }^{[43]}$ 通过 Schiff 碱缩合成环作用, 从 $N, N^{\prime}$-(6-胺基-2-吡啶基)-1,3二甲酰亚胺苯和 5,5'-亚甲基双水杨醛反应制备得到同 时含间苯二甲酰胺单元以及酚环的 $[1+1]$ Schiff 碱大环 24. 采用 ${ }^{1} \mathrm{H} N M R 、 F A B M S$ 和元素分析等手段对大环进 行了表征. 通过 X 射线单晶衍射分析了大环的晶体结 构, 结果表明大环分子具有折叠钳式结构, 在晶态结构 中大环分子间通过氢键, $\pi \cdots \pi$ 堆积作用自组装为具有 1D 槽的柱阵列结构. 同时还通过紫外-可见吸收光谱 滴定技术对大环与系列阴离子的键合作用考察，结果表 明大环 24 对 $\mathrm{H}_{2} \mathrm{PO}_{4}^{-}, \mathrm{HP}_{2} \mathrm{O}_{4}^{-}$具有识别作用. 进一步运 用紫外-可见吸收光谱、核磁和等温量热滴定等技术对 主客体识别行为进行了考察. 结果表明, 大环 24 与 $\mathrm{H}_{2} \mathrm{PO}_{4}{ }^{-}$形成键合比为 $1: 1$ 的配合物, 与 $\mathrm{HP}_{2} \mathrm{O}_{4}{ }^{-}$形成键 合比为 $2: 1$ 的夹心式配合物结构.

受到 Leigh 小组 ${ }^{[4]}$ 合成的含有酰胺键大环分子的影 响, 2019 年, María 课题组 ${ }^{[45]}$ 以二酰氯 25a 与双芐基胺 
<smiles></smiles>

25b 作为反应底物, 通过高度稀释法合成含有四个酰胺 键的大环化合物 25, 产率仅有 $8 \%$ (Scheme 8). 通过核 磁共振氢谱以及高分辨质谱证实了得到的是 $[2+2]$ 构型 的产物. 该小组还在二甲基亚砜作为溶剂中培养得到了 大环分子的单晶, 从单晶结构中可以看出, 两个溶剂分 子进入了大环分子的空腔内部, 通过分子间的氢键结合 形成较为稳定的结构.

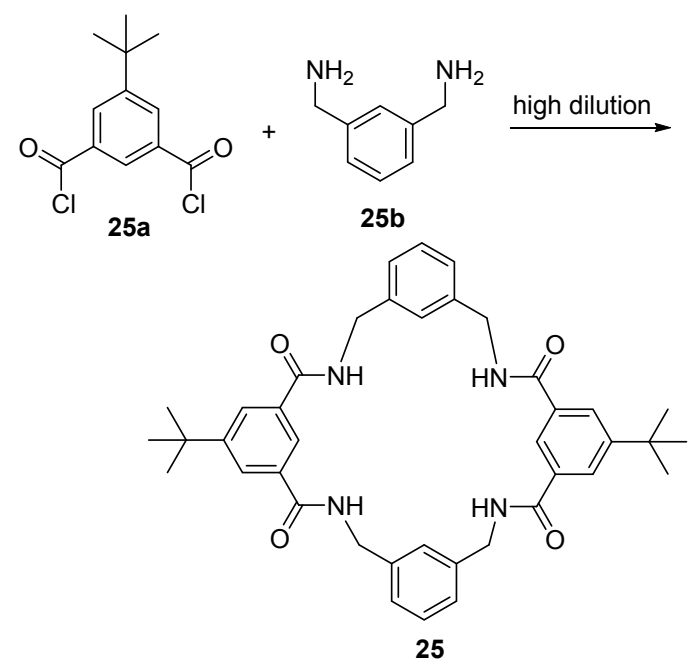

图式 8 大环化合物 $\mathbf{2 5}$ 的合成

Scheme 8 Synthesis of the macrocycle 25

\section{3 展望}

鉴于氢键供体基团构筑大环化合物在分子识别、超 分子催化、自组装方面有许多新颖的特性, 许多新型的 含有氢键供体基团(嫝基、酰胺基以及胺基)被逐渐报道 出来. 因这类大环分子的空腔内部具有氢键供体基团, 通过研究可以模拟生命体内的分子识别行为, 因此对此 类大环化合物的研究一直方兴未艾. 然而目前合成的含 有氢键供体基团构筑大环化合物大多合成路线较长, 产 率较低, 且水溶性较差. 如何通过改变有机合成路线方 法来提高产率以及增加大环分子的水溶性, 将是此类大 环化合物在合成研究中的主要方向. 同时, 在水相环境 中对具有独特性能的客体分子(药物分子、糖类、有机 环境污染物)的识别, 自组装以及超分子催化反应将成 为超分子化学研究的热点内容之一.

\section{References}

[1] (a) Liu, Y.; You, C. C.; Zhang, H. Y. Supramolecular ChemistryMolecular Recognition and Self-Assembly of Synthetic Receptors, Nankai University Press, Tianjin, 2003 (in Chinese).

(刘育, 尤长城, 张衡益, 超分子化学一一合成受体的分子识别 与组装, 天津, 南开大学出版社, 2003.)

(b) Luo, Q. H. Macrocyclic Chemistry -Host-Guest Compound and Supramolecular, Science Press, Beijing, 2009 (in Chinese). (罗勤慧, 大环化学——主一客体化合物和超分子, 科学出版社, 北京, 2009.)

[2] Pedersen, C. J. J. Am. Chem. Soc. 1967, 89, 7017.

[3] Huang, G.-B.; Jiang, W. Prog. Chem. 2015, 27, 744 (in Chinese) (黄国保, 蒋伟, 化学进展, 2015, 27, 744.)

[4] (a) Zheng, B.; Wang, F.; Dong, S.-Y.; Huang, F.-H. Chem. Soc. Rev. 2012, 5, 1621 .

(b) Cheng, H.-B.; Zhang, H.-Y.; Liu, Y. J. Am. Chem. Soc. 2013, $135,10190$.

[5] (a) Liu, Y.; Chen, Y. Acc. Chem. Res. 2006, 10, 681. (b) Chen, Y.; Liu, Y. Chem. Soc. Rev. 2010, 39, 495.

[6] (a) Guo, D.-S.; Wang, K.; Liu, Y. J. Inclusion Phenom. Macrocyclic Chem. 2008, 1-2, 1 .

(b) Guo, D.-S.; Liu, Y. Chem. Soc. Rev. 2012, 41, 5907.

[7] (a) Xue, M.; Yang, Y.; Chi, X.; Zhang, Z.; Huang, F. Acc. Chem. Res. 2012, 45, 1294

(b) Cragg, P. J.; Sharma, K. Chem. Soc. Rev. 2012, 41, 597.

(c) Ogoshi, T.; Yamagishi, T.-A. Eur. J. Org. Chem. 2013, 15, 2961.

(d) Zhang, H.; Zhao, Y. Chem.-Eur. J. 2013, 19, 16862.

(e) Wang, M.-X. Chem. Commun. 2008, 4541.

(f) Guo, Q.-H.; Fu, Z.-D.; Zhao, L.; Wang, M.-X. Angew. Chem., Int. Ed. 2014, 53, 13548.

(g) Wang, M.-X. Acc. Chem. Res. 2012, 45, 182.

(h) Chen, H.; Fan, J.; Hu, X.; Ma, J.; Wang, S.; Li, J.; Yu, Y.; Jia, X.; Li, C. Chem. Sci. 2015, 6, 197.

(i) Tian, X. H.; Chen, C. F. Org. Lett. 2010, 12, 524

(j) Xue, M.; Chen, C. F. Org. Lett. 2009, 11, 5294.

(k) Wang, J. H.; Feng, H. T.; Zheng, Y. S. Chem. Commun. 2014 50, 11407.

(1) Chun, Y.; Singh, N. J.; Hwang, I. C.; Lee, J. W.; Yu, S.-U.; Kim, K.-S. Nat. Commun. 2013, 4, 1797.

(m) Lee, S.; Chen, C.-H.; Flood, A.-H. Nat. Chem. 2013, 5, 704.

[8] Shimizu, L. S.; Smith, M. D.; Hughes, A. D.; Shimizu, K. D. Chem. Commun. 2001, 1592.

[9] (a) Shimizu, L. S.; Hughes, A. D.; Smith, M. D.; Davis, M. J.; Zhang, P.; Loye zur, H.; Shimizu, K. D. J. Am. Chem. Soc. 2003, 125, 14972.

(b) Yang, J.; Dewal, M. B.; Shimizu, L. S. J. Am. Chem. Soc. 2006, 128,8122 .

(c) Yang, J.; Dewal, M. B.; Profeta, S.; Smith, M. D.; Li, Y.; Shimizu, L. S. J. Am. Chem. Soc. 2008, 130, 612.

(d) Dewal, M. B.; Xu, Y.; Yang, J.; Mohammed, F.; Smith, M. D.; Shimizu, L. S. Chem. Commun. 2008, 3909.

(e) Yang, J.; Dewal, M. B.; Sobransingh, D.; Smith, M. D.; Xu, Y.; Shimizu, L. S. J. Org. Chem. 2009, 74, 102.

(f) Xu, Y.; Smith, M. D.; Krause, J.; Shimizu, L. S. J. Org. Chem. 2009, 74, 4874.

(f) Roy, K.; Wang, C.; Smith, M.-D.; Pellechia, P.; Shimizu, L. S. J. Org. Chem. 2010, 75, 5453.

[10] (a) Meshcheryakov, D.; Arnaud-Neu, F.; Böhmer, V.; Bolte, M.; Cavaleri, J.; Hubscher-Bruder, V.; Thondorf, I.; Weener, S. Org. Biomol. Chem. 2008, 6, 3244.

(b) Meshcheryakov, D.; Böhmer, V.; Bolte, M.; Hubscher-Bruder, V.; Arnaud-Neu, F. Chem. Eur. J. 2009, 15, 4811

[11] Dawn, S.; Dewal, M. B.; Sobransingh, D.; Paderes, M. C.; Wibowo, A.; Smith, M.; Krause, J.; Pellechia, P.; Shimizu, L. S. J. Am. Chem. Soc. 2011, 133, 7025.

[12] Roy, K.; Wang, C.; Smith, M. D.; Dewal, M. B.; Wibowo, A. C.; Brown, J. C.; Ma, S.; Shimizu, L. S. Chem. Commun. 2011, 47, 
277.

[13] Geer, M. F.; Smith, M. D.; Shimizu, L. S. CrystEngComm 2011, 13, 3665.

[14] Roy, K.; Wibowo, A.; Pellechia, P.; Ma, S.; Geer, M.; Shimizu, L. S. Chem. Mater. 2012, 24, 4773

[15] Geer, M. F.; Walla, M.; Solntsev, K.; Strassert, C.; Shimizu, L. S. J. Org. Chem. 2013, 78, 5568.

[16] Xiao, T.; Li, S.; Zhang, X.; Lin, C.; Wang, L. Y. Chin. J. Chem. 2013, 31, 627

[17] Zhang, D. S.; Chen, J. P.; Zeng, Y.; Yu, T. J.; Li, Y. Chin. J. Org. Chem. 2013, 33, 110 (in Chinese). (张读山，陈金平，曾毅，于天君，李嫕，有机化学，2013，33, 110.)

[18] Kretschemer, C.; Dittmann, G.; Beck, J. Beilstein J. Org. Chem. 2014, 10,1834

[19] Huang, G. B.; He, Z.; Cai, C.; Pan, F.; Yang, D.; Rissanen, K.; Jiang, W. Chem. Commun. 2015, 51, 15490.

[20] Huang, G. B.; Valkonen, A.; Rissanen, K.; Jiang, W. Chem. Commun. 2016, 52, 9078.

[21] Huang, G. B.; Liu, V.; Valkonen, A.; Yao, H.; Rissanen, K.; Jiang, W. Chin. Chem. Lett. 2018, 29, 91.

[22] (a) Kondo, S.; Sonoda, H.; Katsu, T.; Unno, M. Sens. Actuators, B 2011, 160, 684 .

(b) Satake, A.; Ishizawa, Y.; Katagiri, H.; Kondo, S. J. Org. Chem. 2016, 81,9848 .

[23] Osawa, K.; Tagaya, H.; Kondo, S. J. Org. Chem. 2019, 84, 6623.

[24] Tromans, R.; Carter, T.; Chabanne, T.; Crump, M.; Li, H.; Matlock, J.; Orchard, M.; Davis, A. P. Nat. Chem. 2019, 11, 52.

[25] (a) Shorthill, B. J.; Avetta, C. T.; Glass, T. E. J. Am. Chem. Soc. 2004, 126,12732

(b) Sharma, S. K.; Upreti, S.; Gupta, R. Eur. J. Inorg. Chem. 2007, 3247

(c) Gasparrini, F.; Pierini, M.; Villani, C.; Filippi, A.; Speranza, M. J. Am. Chem. Soc. 2008, 130, 522 .

(d) Shang, X. F.; Lin, H.; Cai, Z. S.; Lin, H. K. J. Heterocycl. Chem. 2008, 45, 1329 .

(d) Ghorai, A.; Gayen, A.; Kulsi, G.; Padmanaban, E.; Laskar, A.; Achari, B.; Mukhopadhyay, C.; Chattopadhyay, P. Org. Lett. 2011, 13,5512 .

[26] (a) Fuller, A. M.; Leigh, D. A.; Lusby, P. J.; Oswald, I. D. H.; Parsons, S.; Walker, D. B. Angew. Chem. 2004, 116, 4004.

(b) Leigh, D. A.; Venturini, A.; Wilson, A. J.; Wong, J. K. Y.; Zerbetto, F. Chem.-Eur. J. 2004, 10, 4960

(c) Fuller, A. M.; Leigh, D. A.; Lusby, P. J.; Oswald, I. D. H.; Parsons, S.; Walker, D. B. J. Am. Chem. Soc. 2005, 137, 12612.

(d) Crowley, J. D.; Leigh, D. A.; Lusby, P. J.; McBurney, R. T.; Perret-Aebi, L. E.; Petzold, C.; Slawin, A. M. Z.; Symes, M. D. J. Am. Chem. Soc. 2007, 129, 15085.

(e) Barrell, M. J.; Leigh, D. A.; Lusby, P. J.; Slawin, A. M. Z. Angew. Chem., Int. Ed. 2008, 47, 8036.

(f) Altieri, A.; Aucagne, V.; Carrillo, R.; Clarkson, G.; D’Souza, D. M.; Dunnet, J.; Leigh, D. A.; Mullen, K. M. Chem. Sci. 2011, 2, 1922.

[27] (a) Klein, E.; Ferrand, Y.; Auty, E. K.; Davis, A. P. Chem. Commun. 2007, 2390.

(b) Ferrand, Y.; Crump, M. P.; Davis, A. P. Science 2007, 318, 619.

(c) Klein, E.; Ferrand, Y.; Barwell, N. P.; Davis, A. P. Angew. Chem., Int. Ed. 2008, 48, 2693.

(d) Challinor, L.; Klein, E.; Davis, A. P. Synlett 2008, 14, 2137.

[28] Qin, B.; Chen, X. Y.; Fang, X.; Shu, Y. Y.; Yip, Y. K.; Yan, Y.; Pan, S. Y.; Ong, W. Q.; Ren, C. L.; Su, H. B.; Zeng, H. Q. Org. Lett. 2008, 10, 5127 .

[29] Qin, B.; Ren, C.; Ye, R.; Sun, C.; Chalid, K.; Chen, X.; Li, Z.; Xue, F.; Su, H.; Chass, G.; Zeng, H. Q. J. Am. Chem. Soc. 2010, 132, 9564.

[30] (a) Helsel, A. J.; Brown, A. L.; Yamato, K.; Feng, W.; Yuan, L. H.;
Clements, A.; Harding, S. V.; Szabo, G.; Shao, Z. F.; Gong, B. J. Am. Chem. Soc. 2008, 130, 15784.

(b) Wang, X.; Liu, R.; Sathyamoorthy, B.; Yamato, K.; Liang, G.; Shen, L.; Ma, S.; Sukumaran, D.; Szyperski, T.; Fang, W.; He, L.; Chen, X.; Gong, B. J. Am. Chem. Soc. 2015, 137, 5879.

[31] Yang, Y.; Feng, W.; Hu, J.; Zou, S.; Gao, R.; Yamato, K.; Kline, M.; Cai, Z.; Gao, Y.; Wang, Y.; Li, Y.; Yang, Y.; Yuan, L.; Zeng, X.; Gong, B. J. Am. Chem. Soc. 2011, 133, 18590.

[32] (a) Ferrand, Y.; Klein, E.; Barwell, N. P.; Crump, M. P.; J. Vicent, J. C.; Boons, G.-J.; Ingale, S.; Davis, A. P. Angew. Chem., Int. Ed. 2009, 49, 1775.

(b) Davis, A. P. Org. Biomol. Chem. 2009, 7, 3629.

(c) Barwell, N. P.; Crump, M. P.; Davis, A. P. Angew. Chem., Int. Ed. 2009, 48, 7363.

[33] Barwell, N. P.; Davis, A. P. J. Org. Chem. 2011, 76, 6548.

[34] (a) Sookcharoenpinyo, B.; Klein, E.; Ferrand, Y.; Walker, B.; Brotherhood, P.; Ke, C.; Crump, M. P.; Davis, A. P. Angew. Chem., Int. Ed. 2012, 51, 4586.

(b) Ke, C.; Destecroix, H.; Crump, M. P.; Davis, A. P. Nat. Chem. 2012, 4, 718

(c) Howgego, J.; Butts, C.; Crump, M. P.; Davis, A. P. Chem. Commun. 2013, 49, 3110.

(d) Destecroix, H.; Renney, C.; Mooibroek, T.; Carter, T.; Stewart, P.; Crump, M. P.; Davis, A. P. Angew. Chem. Int. Ed. 2015, 54, 2057.

(e) Rios, P.; Carter, T.; Mooibroek, T.; Crump, M. P.; Lisbjerg, M.; Pittelkow, M.; Supekar, N.; Boons, G.; Davis, A. P. Angew. Chem., Int. Ed. 2016, 55, 3387.

(f) Carter, T.; Mooibroek, T.; Stewart, P.; Crump, M. P.; Galan, M.; Davis, A. P. Angew. Chem., Int. Ed. 2016, 55, 9311.

(g) Mandal, P.; Kauffmann, B.; Destecroix, H.; Ferrand, Y.; Davis, A. P.; Huc, I. Chem. Commun. 2016, 52, 9355.

(h) Rios, P.; Mooibroek, T.; Carter, T.; Willams, C.; Wilson, M.; Crump, M. P.; Davis, A. P. Chem. Sci. 2017, 8, 4056.

[35] Chen, M. J.; Han, S. J.; Jiang, L. S.; Zhou, S. G.; Jiang, F.; Xu, Z. K.; Liang, J. D.; Zhang, S. H. Chem. Commun. 2010, 46, 3932.

[36] Yang, D. K.; Zeng, Z. J.; Chen, M. J.; Pan, S. W.; Yang, Y.; Li, M.; Lei, C. Y.; Jiang. L. S. Acta Chim. Sinica 2012, 74, 1385 (in Chinese).

(杨登科，曾志坚，陈木娟，潘绍武，杨宇，李媚，雷春燕. 蒋腊 生 化学学报, 2012, 74, 1385.)

[37] Wang, D.; You, L.; Wang, J.; Wang, H.; Zhang, D.; Li, Z. T. Tetrahedron Lett. 2013, 54, 6967.

[38] Chen, Y.; Wang, L.; Zhang, L.; Zhu, J.; Wang, H.; Zhang, D.; Li, Z. T. Tetrahedron 2014, 70, 5483

[39] Huang, G.; Wang, S.; Ke, H.; Yang, L.; Jiang, W. J. Am. Chem. Soc. 2016, 138,14550

[40] Martí-Centelles, V.; Burguete, M. I.; Luis, S. J. Org. Chem. 2016, $81,2143$.

[41] Mao, L.; Pan, W.; Fu, Y.; Chen, L.; Xu, M.; Ren, Y.; Feng, W.; Yuan, L. Org. Lett. 2017, 19, 18.

[42] Wang, F. F.; Ou, M.; Deng, Y. X.; Ran, X.; Zhang, Q. L.; Zhu, B. X. Chin. J. Org. Chem. 2014, 34, 334 (in Chinese). (王芳芳, 欧敏, 邓雅欣, 再旭, 张奇龙, 朱必学, 有机化学, 2014, 34, 334.)

[43] Wei, X. K.; Gu, J. C.; Liu, X. L.; Huang, C.; Zhu, B. X. Chin. J. Org. Chem. 2018, 38, 3386 (in Chinese). (魏小康, 谷静池, 刘兴丽, 黄超, 朱必学, 有机化学, 2018, 38, 3386.)

[44] (a) Johnston, A. G.; Leigh, D. A.; Pritchard, R. J.; Deegan, M. D. Angew. Chem., Int. Ed. Engl. 1995, 34, 1209.

(b) Leigh, D. A.; Venturini, A.; Wilson, A. J.; Wong, J. K. Y.; Zerbetto, F. Chem.-Eur. J. 2004, 10, 4960.

[45] María, D.; Claramunt, R.; Torralba, M.; Torres, M.; Elguero, J. Tetrahedron Lett. 2019, 60, 1206. 